\title{
Hierarchical construction of multi-star small-world networks for real-world applications
}

\author{
Hidefumi Sawai* \\ National Institute of Information and Communications Technology, Nukui-Kitamachi, Koganei, Tokyo, Japan
}

Received: July 24, 2014

Accepted: August 25, $2014 \quad$ Online Published: September 17, 2014

DOI: $10.5430 /$ air.v3n4p1

URL: http://dx.doi.org/10.5430/air.v3n4p1

\begin{abstract}
We proposed a new Small-World network (called n-Star network in which average path-length $L$ becomes absolutely small) inspired by ants' collective behavior. As one of the real-world applications using this network, it is shown that reorganization of the world airline network is possible in the next generation. In addition, it not only has the characteristic that is more immune from random failure and resilient to targeted attacks than bimodal degree distribution network and scale-free network, but also it can maintain Small-World characteristics even when probability of failure is considerably large. Furthermore, the n-Star network can be extended to various types of hierarchical networks, and we performed theoretical analysis of each network structure and derived formulas using various network parameters such as average degree $\langle k\rangle$, average path-length $L$, clustering coefficient $C$ and newly analyzed assortativity (degree correlation) $r$ with the number of star nodes $n$, their peripheral nodes $N_{0}$, the total number of nodes $N$ and the level of hierarchy $l$. We newly discuss the merit and demerit on the current airline network and an airline network based on the n-Star network, and propose a hierarchical architecture of airline network more suitable for real world than both the current airline network and the basic (non-hierarchical) n-Star-based airline network.
\end{abstract}

Key Words: Small-World, Airline network, Hierarchy, Scale-free network, Multi-star network, Complex network

\section{Introduction}

Small-World ${ }^{[1]}$ network has a shorter path-length along a shortcut than those along the other regular paths when the link exchange probability $\rho$ is small enough, however, its average path-length $L$ is equivalent to that of a random graph as a whole. The author got a hint from ants' collective behavior, and proposed a new algorithm to design a new small-world network by searching for the shortest paths and changing the topology of a random graph repeatedly based on the pheromone trail of ants. As a result, the generated network became the multi-star network called n-Star network $^{[2]}$ in which various kinds of network parameters can be analyzed theoretically. It is proved that this network has the shortest average path-length $L$ for a given average degree $\langle k\rangle$ among complex networks through the theoretical analysis and experimental simulation. ${ }^{[2]}$ This fact can also be proved that by defining a new measure of small-world, or the degree of small-worldness $R$, the $R$ value always shows the invariant value 2 for the $\mathrm{n}$-Star networks as small as that of a complete graph with $R \equiv L+p=2$ because $L=1$ and $p=1$ where $p=\langle k\rangle /(N-1)$ is link probability. In addition, we examined a method for reorganizing the nextgeneration world airline network to apply this network to real world using real data such as the latitude and longitude of the world-ranking major cities, population and the distance between the major cities along the geodesic line of Earth. ${ }^{[3]}$ Compared to a current airline network, the nStar network-based airline network was found to be more efficient in terms of average cruising distance $L$ (dist) and average number of transits $L(s t e p)$ (it can reduce at least $20 \%-30 \%$ in a relatively small domain of link probability $p=\langle k\rangle /(N-1)$, where $\langle k\rangle$ is the average number of different flight routes per airport, and $N$ is the total number of airports). ${ }^{[3]}$ Furthermore, we showed the n-Star network is immune from random failure on nodes and links, and resilient to targeted attacks on star nodes even if all star nodes

\footnotetext{
*Correspondence: Hidefumi Sawai; Email: sawai@nict.go.jp; Address: National Institute of Information and Communications Technology 4-2-1, Nukui-Kitamachi, Koganei, Tokyo 184-8795 Japan.
} 
fail by the targeted attacks, a self-organizing algorithm can be applied automatically to recover the original topology especially in a mobile communication environment. ${ }^{[4]}$

In this study, we show the n-Star network can be constructed to several hierarchical types of architecture: hierarchical type, torus type, star type, star-torus type, fractal type I and fractal type II. Their network parameters such as average degree $\langle k\rangle$, average path-length $L$, clustering coefficient $C$, degree of small-worldness $R^{[5]}$ and assortativity $r^{[6]}$ can be analyzed theoretically with the relationship of $n, N_{0}, N$ and $l$ where $n$ is the number of star nodes, $N_{0}$ is the number of peripheral nodes, $N$ is the total number of nodes and $l$ is the level of hierarchy. As one of real-world applications, a new hierarchical airline network is proposed using one of the best hierarchical architectures of n-Star network.

This article constitutes as follows: in the next Section 2, we describe the method for emerging a new small-world network inspired by ants' collective behavior. Section 3 describes the extension of n-Star network to six kinds of hierarchical structures. Section 4 describes analysis on the hierarchical structures from the viewpoints of small-world and assortativity (i.e. degree correlation). Section 5 describes a real-world application in the field of logistics using one of the hierarchical structures. Experimental results and Discussions are in Section 6. Finally, we summarize the contents in Conclusion.

\section{Emerging new small-world}

Several methods to emerge small-world networks have been proposed. One type of method is based on simple iteration algorithms with growing vertices and edges of a network. $^{[7-13]}$ Another type of method is based on rewiring edges while keeping a total number of nodes in a network, $^{[14]}$ a connected lattice ${ }^{[15]}$ and a small-world network. ${ }^{[16]}$ A study on the relative tradeoff between wiring and connectivity has been reported ${ }^{[17]}$ when the network seeks to minimize wiring, a regular graph results, and at the other extreme, when connectivity is maximized, a random network is obtained. Unlike the model of Watts and Strogatz, ${ }^{[18]}$ they find an alternate route to small-world behavior through the formation of hubs, small clusters where one vertex is connected to a large number of neighbors. A study on seeking an efficient small-world network results in the network with several centers and a connected subnetwork of shortcuts. ${ }^{[19]}$ Furthermore, small-world behavior in time-varying graphs are also recently investigated. ${ }^{[20]}$

A method for creating new small-world, called n-Star network, inspired by ants' collective behavior is described in the literature. ${ }^{[2]}$ This network holds a large clustering coefficient $C$ and the shortest average path-length $L$ (these properties satisfy the definition of small-world ${ }^{[1]}$ ) among conventional complex networks.

We present the results of a theoretical analysis for the multistar network architecture. When the number of stars in the multi-star network is $n(n=1,2,3, \cdots, N)$, we can call it "n-Star network". Figure 1 (left) shows the n-Star networks when $n=1,2,3$ and 4; the stars are located at the center with the peripheral links as denoted by the dotted lines. All of the star nodes are completely connected with each other forming a "clique", and all peripheral nodes are connected with all of the star nodes.

For the n-Star networks with the peripheral links ( $m$ : the number of peripheral links, $m=0,1,2, \cdots,{ }_{N-n} C_{2}$ ), the average degree $\langle k\rangle$, average path-length $L$ and clustering coefficient $C$ are calculated as follows:

$$
\begin{aligned}
& \langle k\rangle=\frac{n(2 N-n-1)+2 m}{N} ; n=1,2,3, \cdots, N, m=0,1,2, \cdots,\left(\begin{array}{c}
N-n \\
2
\end{array}\right) . \\
& L=\frac{2 N(N-n-1)+n(n+1)-2 m}{N(N-1)} ; n=1,2,3, \cdots, N, m=0,1,2, \cdots,\left(\begin{array}{c}
N-n \\
2
\end{array}\right) .
\end{aligned}
$$

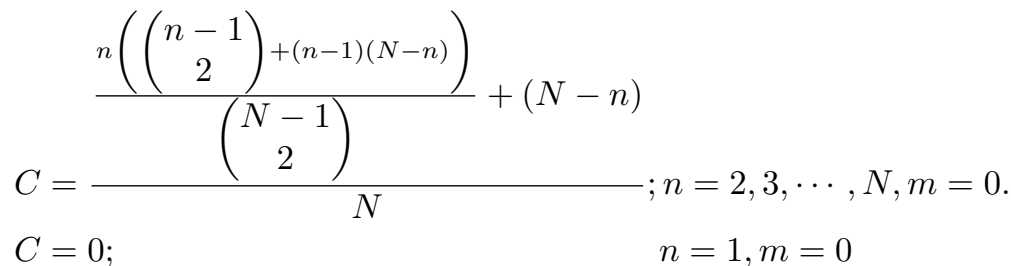

When $n$ is removed using Eqs. (1) and (2), we obtain Eq.(4) as follows:

$$
L=2-\frac{\langle k\rangle}{N-1}=2-p, p=\frac{\langle k\rangle}{N-1},
$$


where $p$ is link probability.

We can obtain the invariant $R \equiv L+p \equiv 2$ directly from Eq.(4) independent of the values of $L$ and $\langle k\rangle$ (hence, independent of $n, m$ and $N)$. This means that the $R$ value is invariant (=2) for the $n$-Star networks. The definition of the small-worldness in this paper is original. However, another different definition of small-worldness was proposed as the ratio $\sigma^{[21]}$ with comparison of clustering coefficient $C_{\text {ran }}$ and average path-length $L_{\text {ran }}$ of a random graph as follows:

$$
\sigma=\gamma / \lambda, \gamma=C_{n e t} / C_{\text {ran }}>1, \lambda=L_{n e t} / L_{\text {ran }}
$$

where $C_{n e t}$ is clustering coefficient and $L_{n e t}$ is average path-length of a network. This definition is a relative combination of clustering coefficient and average path-length of a network relative to those of a random graph. On the other hand, our definition is based on the absolute value $R \equiv L+p$ that simply combined average path-length $L$ (i.e. efficiency of a network) with link probability $p$ (i.e. cost or resource of a network) independent of clustering coefficient $C$ where the $R$ value is always 2 for complete graphs and any single and n-Star networks both with and without peripheral links, and the $R$ values for the other networks always show the values more than 2 . This definition is more universal than the conventional definition of smallworld with a short average path-length $L$ and a large clustering coefficient $C$ because even a single-star network has the small-world property although its clustering coefficient $C$ is zero which is against the conventional definition of smallworld with a large value of $C$.
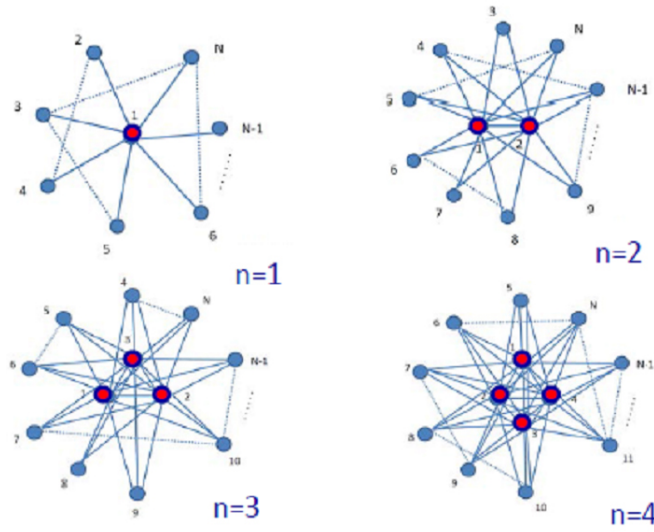

Therefore, we can comparatively evaluate each network as to the degree of small-world based on this invariant $R$. Note that since the $R$ value for complete graphs is 2 because $L=1$ and $p=1$, which implies the $\mathrm{n}$-Star networks are always as small as complete graphs for any given values of $\langle k\rangle$ corresponding to cost of network. Based on this fact, the $R$ value could be regarded as a degree of small-world (we may call it "small-worldness"). As the Barabási-Albert (BA) model ${ }^{[22]}$ possesses a "scale-free" characteristic with the order of $L \sim O(\log N)^{[23]}(O(x)$ means the order of $x)$, the $R$ value is comparable to those in a random graph and the Watts-Strogatz (WS) model. ${ }^{[2]}$ These results indicate that the n-Star networks can always achieve the maximum degree of small-world.

As the n-Star network has the absolutely minimum average path-length $L$ among known complex networks, it can be applicable to search for the minimum paths in the fields of communication and logistics networks, etc., which will be very beneficial for reducing communication and transportation costs effectively while increasing the efficiency at maximum.

We designed a new world airline network using the n-Star network where it could reduce the average cruising distance $L$ (dist) (also the average number of transits $L($ dist $)$ ) as much as $20 \%-30 \%$ compared to a current airline network (the current airline network has a scale-free characteristic that can be modeled by the BA model) for a relatively small average degree $\langle k\rangle$ which is proportional to the number of different flight routes per airport. ${ }^{[3]}$

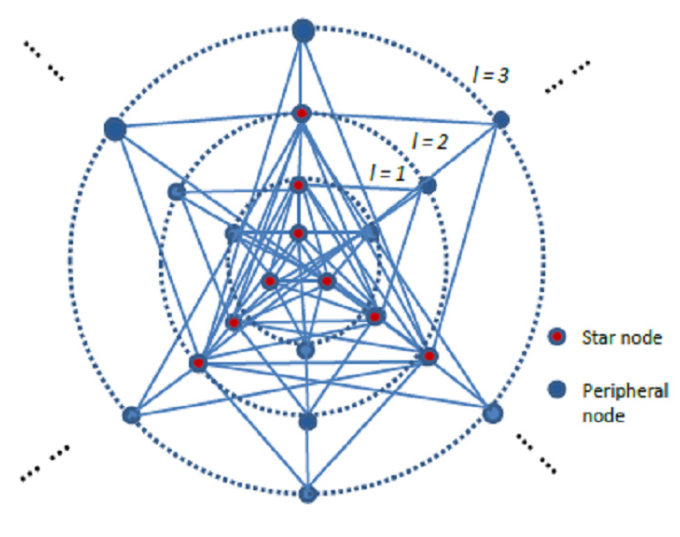

Figure 1: n-Star network architectures having the star nodes at the center (left), and how to build hierarchical structure based on the n-Star networks (right). Some peripheral nodes in the $l$-th layer become the star nodes in the next $(l+1)$-th layer where $l=1,2,3, \cdots, \infty$ for the case of $n=3$.

\section{Hierarchical structure in nature and real world}

Nature contains hierarchical structures in itself from microscale (i.e. elementary particles) to macro-scale (i.e. the Universe). However, mid-scale phenomena play a major role in our daily life. For examples, many kinds of real-world networks such as social networks between humans, WWW (World Wide Web), the Internet, logistics networks such as airline networks, brain networks between neurons, biological networks for metabolism in a cell contain their "scalefree" structures ${ }^{[24-26]}$ and often fractal structures with hierarchy. ${ }^{[27-30]}$ The interaction between the functional nodes 
can be expressed as links in a complex network with hierarchy. The study ${ }^{[31]}$ proposed a geographical scale-free network model with the nodes embedded in a fractal space where the fractal dimension $D$ of the embedding space was found to influence the scale-free exponent $\gamma$. The study ${ }^{[32]}$ discusses on emergence of fractal scale-free networks from stochastic evolution on the Cayley tree. The study ${ }^{[33]}$ analyses mean commute time for random walks on hierarchical scale-free networks called $(x, y)$-flowers.

Although the networks with "scale-free" degree distribution show the small-world characteristics with the average path-length $L \sim O(\log N)$, their network topologies are not necessarily optimized because a random graph also shows a similar property with the average path-length $L \sim$ $O(\log N)$, which implies the efficiency of scale-free networks in terms of average path-length $L$ for a given average degree $\langle k\rangle$ (corresponding to cost) is as same as that of a random graph.

As is described in the previous section, the n-Star network architecture is completely optimized in terms of average path-length $L$ for a given average degree $\langle k\rangle$ or link probability $p$. In other words, the degree of small-world or "small-worldness" $R$ always shows the minimum value of 2 among any kinds of complex networks. Based on this n-Star network architecture, building several kinds of hierarchical structures would provide significant values when we design hierarchical structures of complex networks and apply them to both nature and real world.

The concept of hierarchical structure of n-Star network is shown in Figure 1 (right) where the star nodes on the $l$-th layer are located at the center, and another star nodes on the next $(l+1)$-th layer are located as a part of the peripheral nodes on the $l$-th layer. Namely, some nodes are commonly used as the star nodes on the $(l+1)$-th layer and the peripheral nodes on the $l$-th layer. The level of hierarchy $l$ can increase as $l=1,2,3, \cdots \infty$ which will form a fractal structure.

In the following subsections, six kinds of hierarchical structures of n-Star network are described with their adjacency matrices, and theoretical analysis results on several network parameters such as average degree $\langle k\rangle$, average path-length $L$, clustering coefficient $C$ with 3D viewgraphs and degree distribution $P(k)$ are shown in each Figure.

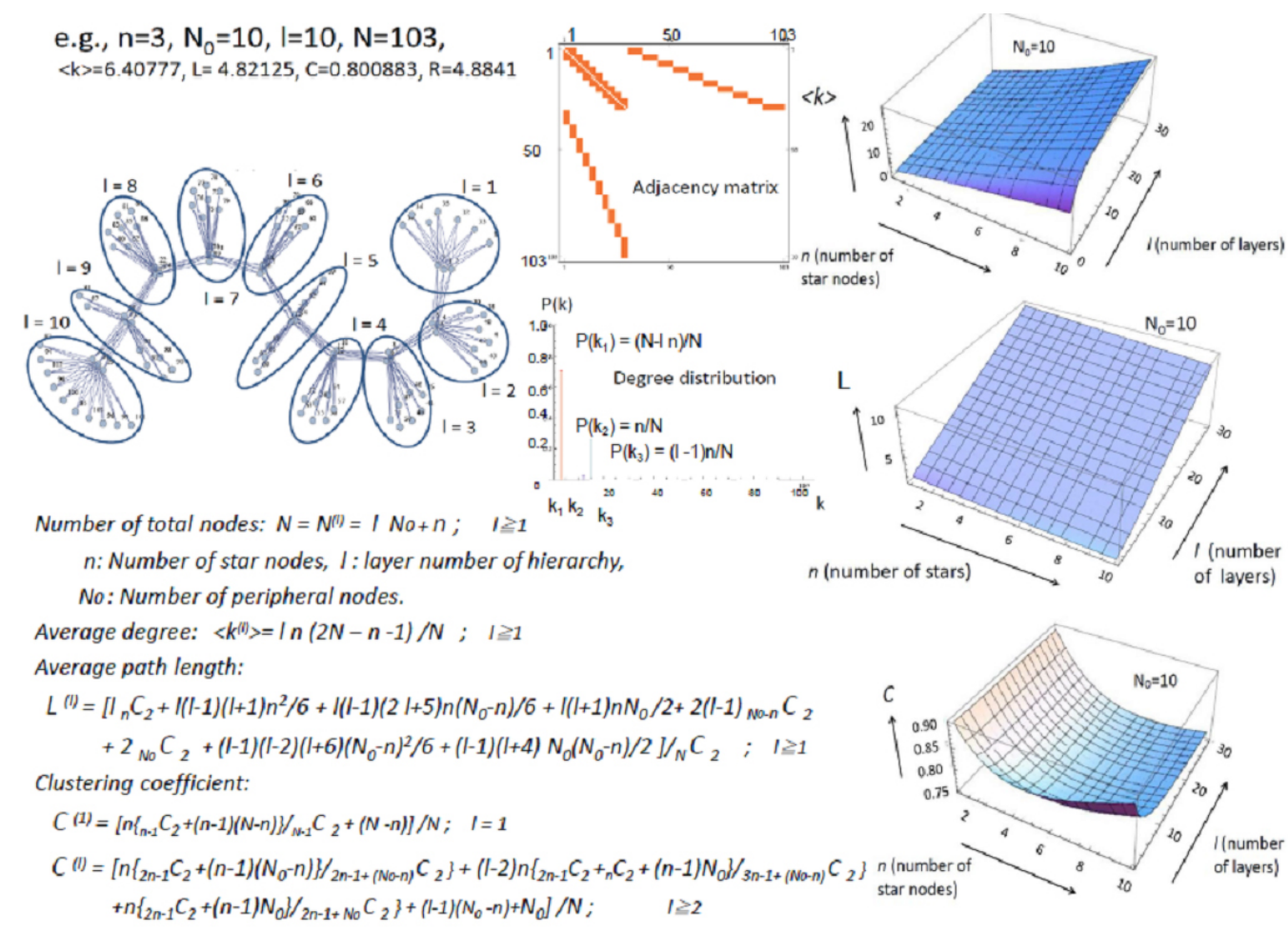

Figure 2: Schematic diagram of the hierarchical type (top-left), its adjacency matrix (top-middle), degree distribution $P(k)$ (middle), the 3D viewgraphs of average degree $\langle k\rangle$ (top-right), average path-length $L$ (middle-right) and clustering coefficient $C$ (bottom-right), and analytical results on several network parameters such as average degree $\langle k\rangle$, average path-length $L$ and clustering coefficient $C$ (bottom-left) are shown.

\subsection{Hierarchical type of $\mathbf{n}$-Star network}

The hierarchical type of $n-S t a r$ network is shown in Figure 2 where the number of star nodes $n=3$, the number of periph- eral nodes $N_{0}=10$, the number of total nodes $N=103$ and the level of hierarchy $l=10$. For this set of parameters, $\langle k\rangle$ $=6.4078, L=4.8213, C=0.8009$ and $R=4.8841$. The con- 
figuration is shown at the top-left, the adjacency matrix and degree distribution at the top-middle, the 3D viewgraphs of average degree $\langle k\rangle$, average path-length $L$ and clustering coefficient $C$ with a relationship of $(n, l)$ are shown from the top-right to bottom-right, respectively. The analytic results on these parameters are shown at the bottom-left. This architecture is the same as that of Figure 1 (right) when $l=$ 10. The average degree $\langle k\rangle$ almost increases in proportion to $n$, and the average path-length $L$ increases in proportion to $l$, the clustering coefficient $C$ keeps its high value of 0.8 . The degree distribution $P(k)$ mainly shows a trimodal manner with the degrees of $k_{1}, k_{2}$ and $k_{3}\left(k_{1}>k_{3}>k_{2}\right)$. The clustering coefficient $C$ at $l=1$ coincides with that of a basic n-Star network, i.e. Eq.(3).

\subsection{Torus type of $\mathbf{n}$-Star network}

The torus type of n-Star network is shown in Figure 3 where the number of star nodes $n=3$, the number of peripheral nodes $N_{0}=10$, the number of total nodes $N=100$ and the level of hierarchy $l=10$. For this set of parameters, $\langle k\rangle=6.6, L=3.9313, C=0.7943$ and $R=3.9980$. Similarly, the configuration is shown at the top-left, the adja- cency matrix and degree distribution at the top-middle, the $3 \mathrm{D}$ viewgraphs of average degree $\langle k\rangle$, average path-length $L$ and clustering coefficient $C$ with a relationship of $(n, l)$ are shown from the top-right to bottom-right, respectively. The analytic results on these parameters are shown at the bottom-left. This network is formed by connecting the highest level of $l$ with the first level of $l=1$ in the hierarchical type of n-Star network shown in Figure 2. This architecture is similar to the "Snake of Uroboros" shown at the center of the configuration which is an analogy of hierarchical nature from elementary particles to the Universe containing elementary particles with different scales. The average degree $\langle k\rangle$ almost increases in proportion to $n$, and the average path-length $L$ increases in proportion to $l$, the clustering coefficient $C$ keeps its high value of 0.79 . The degree distribution $P(k)$ exactly shows a bimodal distribution manner with the degrees of $k_{1}$ and $k_{2}\left(k_{1}>k_{2}\right)$. Note that the formulas of average path-lengths $L$ are different depending on the level of $l$ with even or odd numbers more than 3 , and the clustering coefficients $C$ can be calculated with the level of $l$ more than 3 which enables to make it the torus structure, as shown in Figure 3.
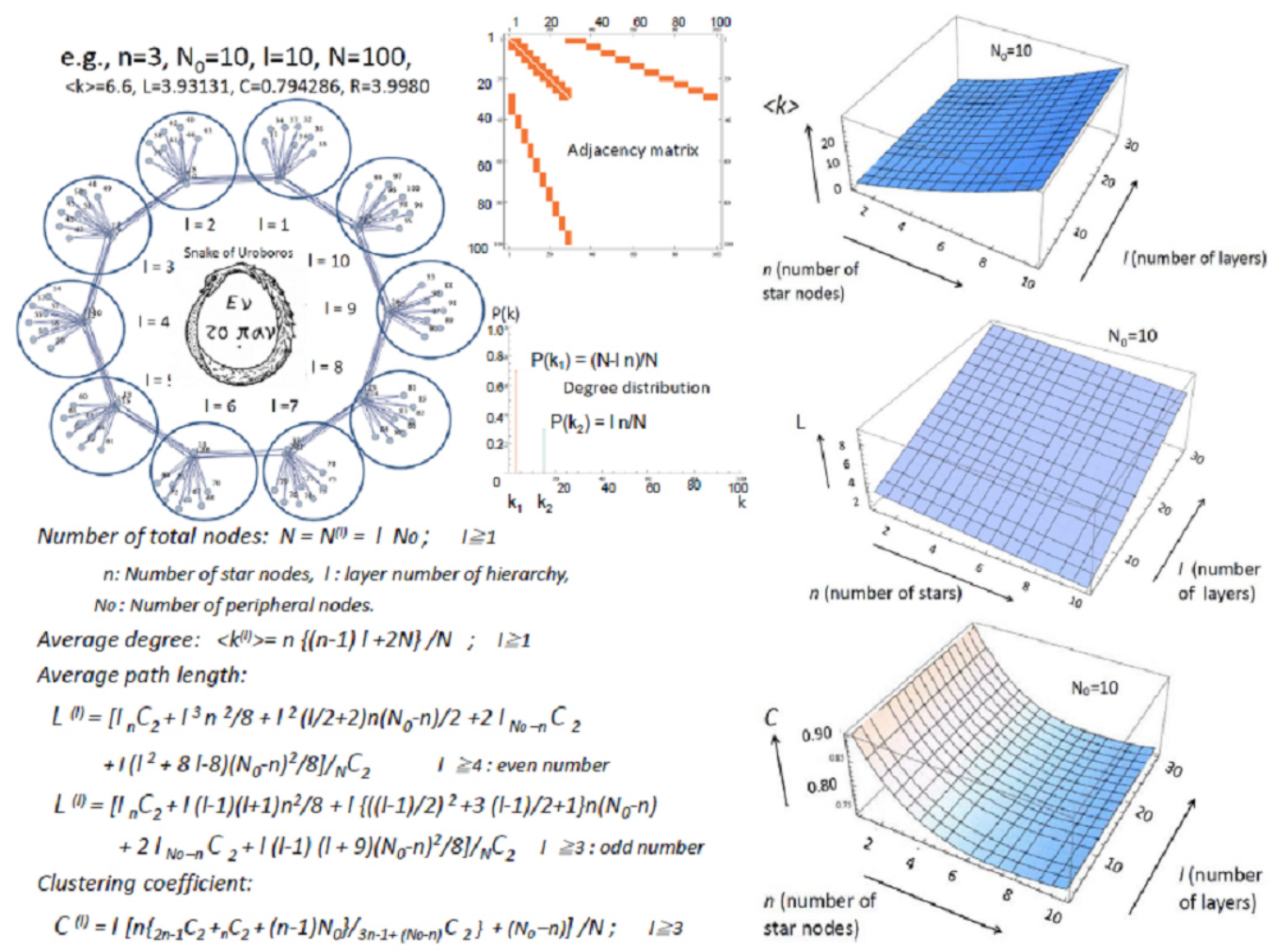

Figure 3: Schematic diagram of the torus type (top-left), its adjacency matrix (top-middle), degree distribution $P(k)$ (middle), the 3D viewgraphs of average degree $\langle k\rangle$ (top-right), average path-length $L$ (middle-right) and clustering coefficient $C$ (bottom-right), and analytical results on several network parameters such as average degree $\langle k\rangle$, average path-length $L$ and clustering coefficient $C$ (bottom-left) are shown. 


\subsection{Star type of $\mathbf{n}$-Star network}

The star type of n-Star network is shown in Figure 4 where the number of star nodes $n=3$, the number of peripheral nodes $N_{0}=7$, the number of total nodes $N=100$ and the level of hierarchy $l=10$. For this set of parameters, $\langle k\rangle=6.42, L=3.042, C=0.8028$ and $R=3.1073$. Similarly, the configuration is shown at the top-left, the adjacency matrix and degree distribution at the top-middle, the $3 \mathrm{D}$ viewgraphs of average degree $\langle k\rangle$, average pathlength $L$ and clustering coefficient $C$ with a relationship of $(n, l)$ are shown from the top-right to bottom-right, respectively. The analytic results on these parameters are shown at the bottom-left. This network is formed by connecting the higher $(l-1)$ clusters with the first level $(l=1)$ cluster at the center. This is a star-like architecture both in each level and as a whole which shows a fractal (self-similar) structure. The average degree $\langle k\rangle$ almost increases in proportion to $\mathrm{n}$, and the average path-length $L$ decreases as $\mathrm{n}$ increases, saturating with the increase in $l$, and the clustering coefficient $C$ keeps its high value of 0.80 . The degree distribution $P(k)$ shows a trimodal manner with the degrees of $k_{1}, k_{2}$ and $k_{3}\left(k_{1}>k_{2}>k_{3}\right)$. Note that the formulas of average path-lengths $L$ and clustering coefficients $C$ are calculated with the level of 1 more than 3 which enables to make it the star structure, as shown in Figure 4.

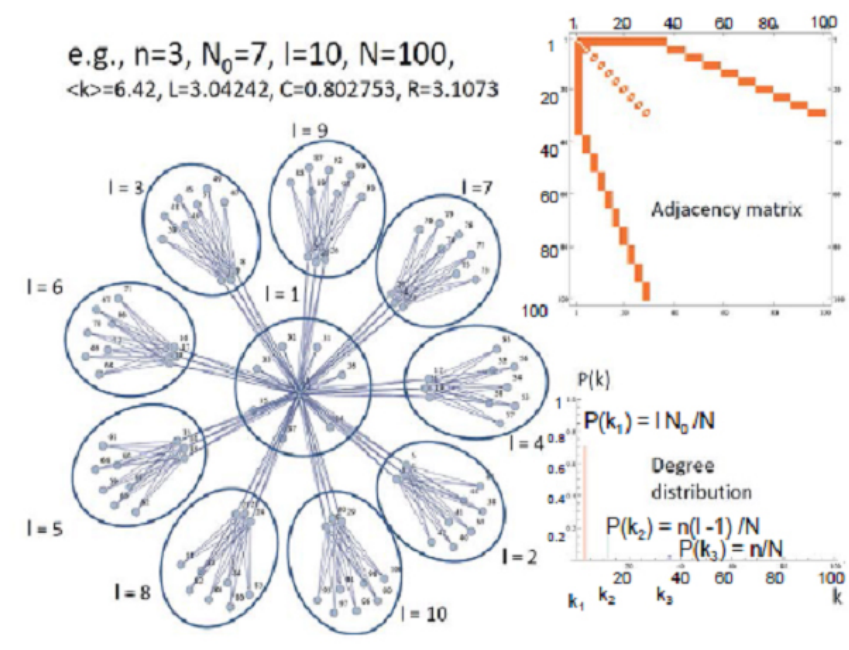

Number of total nodes: $N=N^{(i)}=I(n+N o) ; \quad l \geqq 1$
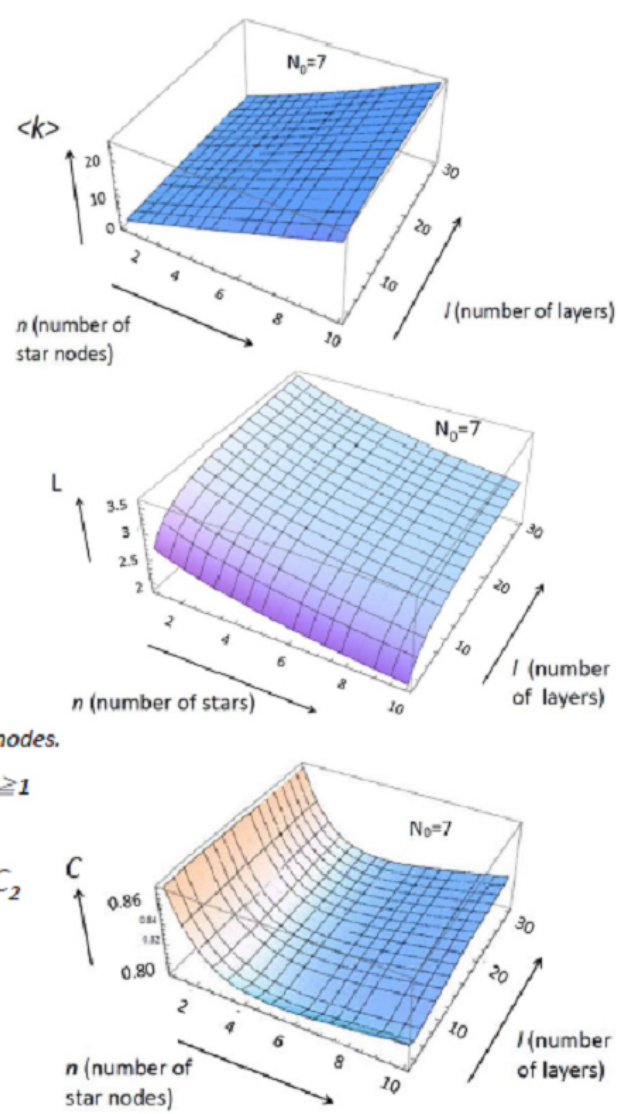

Figure 4: Schematic diagram of the star type (top-left), its adjacency matrix (top-middle), degree distribution $P(k)$ (middle), the 3D viewgraphs of average degree $\langle k\rangle$ (top-right), average path-length $L$ (middle-right) and clustering coefficient $C$ (bottom-right), and analytical results on several network parameters such as average degree $\langle k\rangle$, average path-length $L$ and clustering coefficient $C$ (bottom-left) are shown.

\subsection{Star-Torus type of $\mathbf{n}$-Star network}

The star-torus type of $\mathrm{n}$-Star network is shown in Figure 5 where the number of star nodes $n=3$, the number of peripheral nodes $N_{0}=7$, the number of total nodes $N=100$ and the level of hierarchy $l=10$. For this set of parameters, $\langle k\rangle=8.04, L=2.86061, C=0.8143$ and $R=2.9418$. Similarly, the configuration is shown at the top-left, the adjacency matrix and degree distribution at the top-middle, the
3D viewgraphs of average degree $\langle k\rangle$, average path-length $L$ and clustering coefficient $C$ with a relationship of $(n, l)$ are shown from the top-right to bottom-right, respectively. The analytic results on these parameters are shown at the bottom-left. This network is formed by integrating the torus type in Figure 3 with the star type in Figure 4. This architecture is like a wheel structure. The average degree $\langle k\rangle$ almost increases in proportion to $n$, saturating with the increase in 
$l$, and the average path-length $L$ decreases as $n$ increases, saturating with the increase in $l$, the clustering coefficient $C$ keeps its high value of 0.81 . The degree distribution $P(k)$ shows a trimodal manner with the degrees of $k_{1}, k_{2}$ and $k_{3}$ $\left(k_{1}>k_{2}>k_{3}\right)$. Note that the formulas of average pathlengths $L$ and clustering coefficients $C$ are calculated with the level of $l$ more than 4 which enables to make it the startorus structure, as shown in Figure 5.

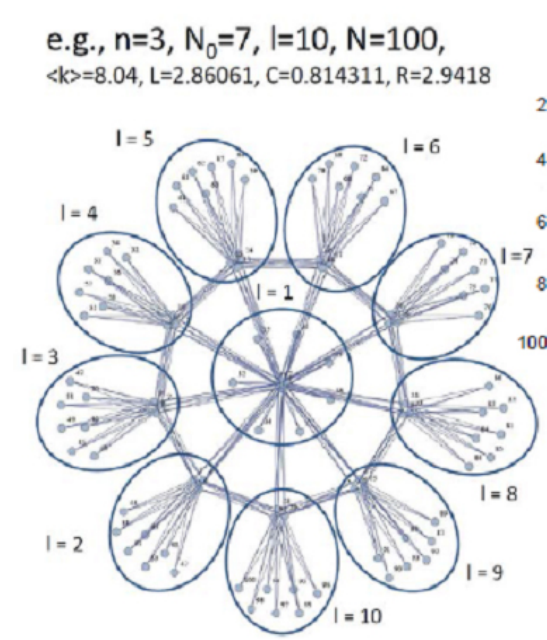

Number of total nodes: $N=N^{(n)}=1(n+N o) ; \quad l \geq 1$

$n$ : Number of star nodes, $1:$ layer number of hierarchy, No: Number of peripheral nodes.

Average degree: $\left\langle k^{(1)}\right\rangle=n\left\{(5 n-1)|-4 n+2| N_{0}\right\} / N ; j \geq 4$

Average path length:

$L(1)=\left[I_{n} C_{2}+2 I_{N_{0}} C_{2}+(l-1)(l-2) n^{2}+\left(3 l^{2}-6 I+4\right) n N_{0}+2(I-1)^{2} N_{0}^{2}\right] /{ }_{N} C_{2}, I \geq 4$

Clustering coefficient:

$C^{(i)}=\left[n\left\{n-1 C_{2}+(1-1)\left(_{n} C_{2}+2 n^{2}-n\right)+(n-1) N_{0}\right\} / 1 n-1+N_{0} C_{2}\right\}$

$\left.+(1-1) n\left\{n-1 C_{2}+3{ }_{n} C_{2}+3 n(n-1)+2 n^{2}+(n-1) N_{a}\right\} / 4 n-1+N_{0} C_{2}+\mid N_{0}\right] / N ; 1 \geq 5$,

$C^{(4)}=\left[n\left\{{ }_{n-1} C_{2}+(1-1)\left(C_{n} C_{2}+2 n^{2}-n\right)+(n-1) N_{0}\right\} / 1 n-1+N_{0} C_{2}\right.$

$\left.+(l-1) n\left\{n-1 C_{2}+3{ }_{n} C_{2}+3 n(n-1)+3 n^{2}+(n-1) N_{a}\right\} / 4 n \cdot 1+N o C_{2}+I N_{0}\right] / N ; \quad l=4$,
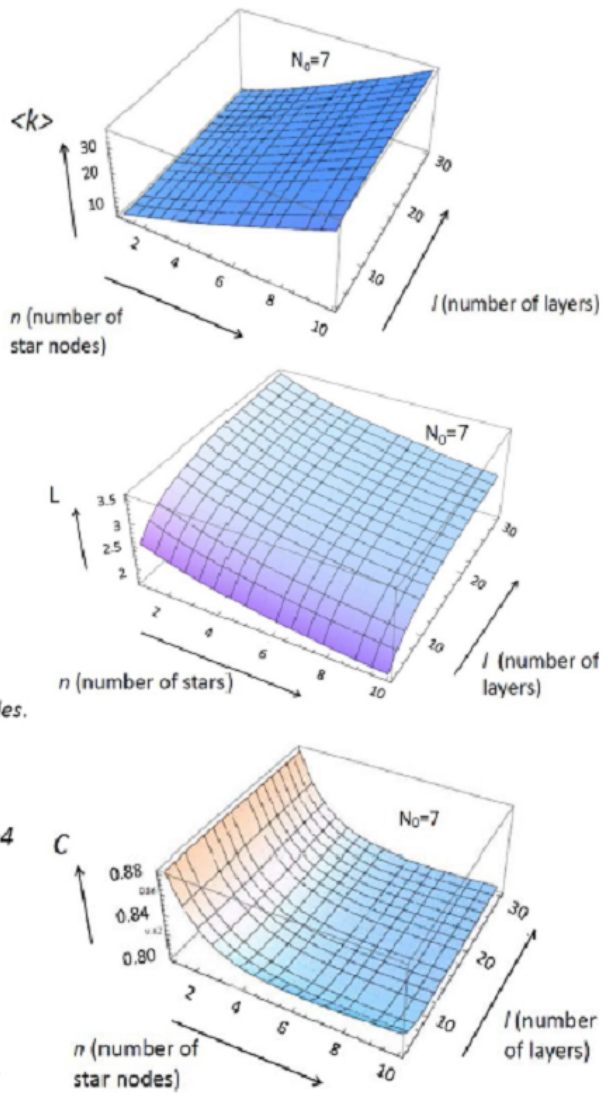

Figure 5: Schematic diagram of the star-torus type (top-left), its adjacency matrix (top-middle), degree distribution $P(k)$ (middle), the 3D viewgraphs of average degree $\langle k\rangle$ (top-right), average path-length $L$ (middle-right) and clustering coefficient $C$ (bottom-right), and analytical results on several network parameters such as average degree $\langle k\rangle$, average path-length $L$ and clustering coefficient $C$ (bottom-left) are shown.

\subsection{Fractal type I of $\mathbf{n}$-Star network}

The fractal type I of n-Star network is shown in Figure 6 where the number of star nodes $n=3$, the number of peripheral nodes $N_{0}=7$, the number of total nodes $N=100$ and the level of hierarchy $l=10$. For this set of parameters, $\langle k\rangle=9.12, L=2.7394, C=0.8277$ and $R=2.8315$. Similarly, the configuration is shown at the top-left, the adjacency matrix and degree distribution at the top-middle, the $3 \mathrm{D}$ viewgraphs of average degree $\langle k\rangle$, average path-length $L$ and clustering coefficient $C$ with a relationship of $(n, l)$ are shown from the top-right to bottom-right, respectively. The analytic results on these parameters are shown at the bottom-left. This network is formed by integrating the $n$ clusters at the center with the $N_{0}$ peripheral clusters which is self-similar (i.e. fractal) to the structure in each cluster with the $n$ star nodes and $N_{0}$ peripheral nodes. The average degree $\langle k\rangle$ almost super-linearly increases as $n$ increases, independent of the increase in $l$, and the average path-length $L$ tends to saturate as $l$ increases, the clustering coefficient $C$ keeps its high value of 0.82 . The degree distribution $P(k)$ shows a trimodal manner with the degrees of $k_{1}, k_{2}$ and $k_{3}$ $\left(k_{1}>k_{2}>k_{3}\right)$. Note that the formulas of average pathlengths $L$ and clustering coefficients $C$ can be calculated with the level of $l$ more than $n$ which enables to make it the fractal type I structure. 

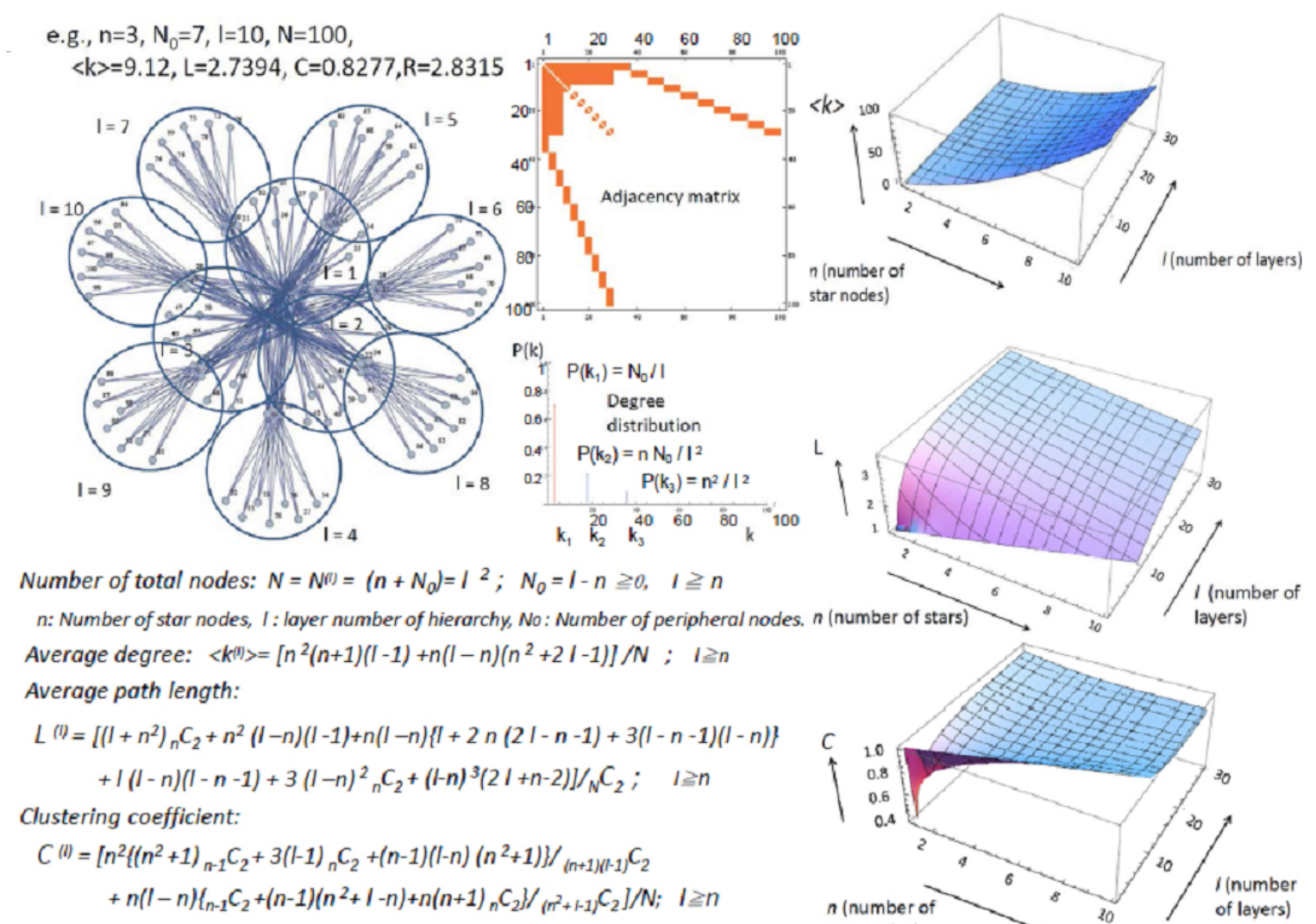

$n:$ Number of star nodes, I: layer number of hierarchy, No: Number of peripher
Average degree: $\left\langle k^{(i)}\right\rangle=\left[n^{2}(n+1)(I-1)+n(I-n)\left(n^{2}+2 I-1\right)\right] / N ; 1 \geq n$ Average path length:

$$
\begin{aligned}
& L(I)=\left[\left(I+n^{2}\right){ }_{n} C_{2}+n^{2}(I-n)(I-1)+n(I-n)\{I+2 n(2 I-n-1)+3(I-n-1)(I-n)\}\right. \\
& \left.+I(I-n)(I-n-1)+3(I-n)^{2}{ }_{n} C_{2}+(I-n)^{3}(2 I+n-2)\right] /{ }_{N} C_{2} ; \quad I \geq n \\
& \text { Clustering coefficient: } \\
& \left.+n(I-n)\left(n-1 C_{2}+(n-1)\left(n^{2}+I-n\right)+n(n+1){ }_{n} C_{2}\right\} /\left(n^{2}+1-1\right) C_{2}\right] / N ; \quad I \geqq n
\end{aligned}
$$

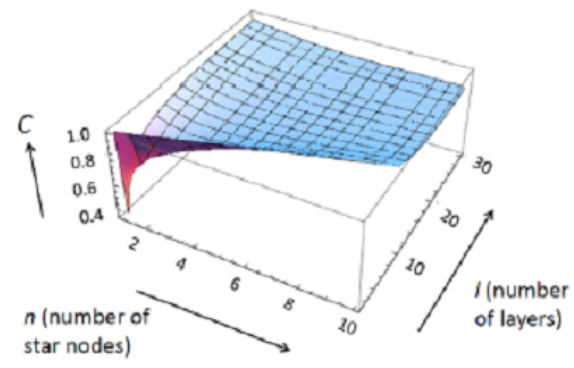

Figure 6: Schematic diagram of the fractal type I (top-left), its adjacency matrix (top-middle), degree distribution $P(k)$ (middle), the 3D viewgraphs of average degree $\langle k\rangle$ (top-right), average path-length $L$ (middle-right) and clustering coefficient $C$ (bottom-right), and analytical results on several network parameters such as average degree $\langle k\rangle$, average path-length $L$ and clustering coefficient $C$ (bottom-left) are shown.

\subsection{Fractal type II of $\mathbf{n}$-Star network}

The fractal type II of n-Star network is shown in Figure 7 for the number of star nodes $n=5$, the number of peripheral nodes $N_{0}=15$, the number of total nodes $N=100$ and the level of hierarchy $l=5$. For this set of parameters, $\langle k\rangle=8.7, L=2.6141, C=0.84142$ and $R=2.7293$. Similarly, the configuration is shown at the top-left, the adjacency matrix and degree distribution at the top-middle, the 3D viewgraphs of average degree $\langle k\rangle$, average path-length $L$ and clustering coefficient $C$ with a relationship of $\left(n, N_{0}\right)$ are shown from the top-right to bottom-right, respectively. The analytic results on these parameters are shown at the bottom-left. This network is formed by integrating the $n$ stars at the center with the $n$ peripheral clusters where each star node is commonly used as each star node at the center as well as in each peripheral cluster. The average degree $\langle k\rangle$ almost linearly increases as $n$ increases, and the average path-length $L$ tends to saturate as $n$ increases, and the clustering coefficient $C$ keeps its high value of 0.84 . The degree distribution $P(k)$ shows a trimodal manner with the degrees of $k_{1}, k_{2}$ and $k_{3}\left(k_{1}>k_{2}>k_{3}\right)$. Note that the formulas of average path-lengths $L$ and clustering coefficients $C$ can be calculated with the level of $l$ always equal to $n$ which enables to make it the fractal type II structure.

\section{Analysis of hierarchical network architec- tures}

\subsection{Analysis of Small-Worldness}

We analyzed several types of the hierarchical structures of n-Star network from the viewpoint of small-worldness $R$. The value $R$ for the basic n-Star network is always 2 independent of network parameters such as $L$ and $p$. If the value $L$ increases, $p$ decreases, and vice versa. For the other kinds of complex networks, the $R$ values are always more than 2 . Therefore, how far the value $R$ for a network is from 2 becomes the measure of small-worldness. The closer the value $R$ becomes to 2, the smaller the network is. By calculating the values $R$ for several kinds of the hierarchical structures shown in Figures from 2 to 7 , the results are shown in Table 1 using an adequate set of network parameters such as $n, N_{0}, N$ and $l$ for a fair comparison with each other. Clustering coefficients $C$ and assortativities $r$ (described in the next subsection) are also shown in this Table. According to the results in Table 1, the degree of small-worldness $R$ increases in the order of entry from the top among all types of 
the hierarchical structures except the basic n-Star network, i.e. the hierarchical type, torus type, star type, star-torus type, fractal type I and fractal type II. Furthermore, the 3D viewgraphs of the values $R$ for all types of the hierarchy are shown in Figure 8 with the combination of $n$ and $l$ except for the case of the fractal type II because $n$ is always equals to $l$ in this case. e.g., $n=5, N_{0}=15, l=5, N=100$,
$\quad\langle k>=8.7, L=2.6141, C=0.841422$

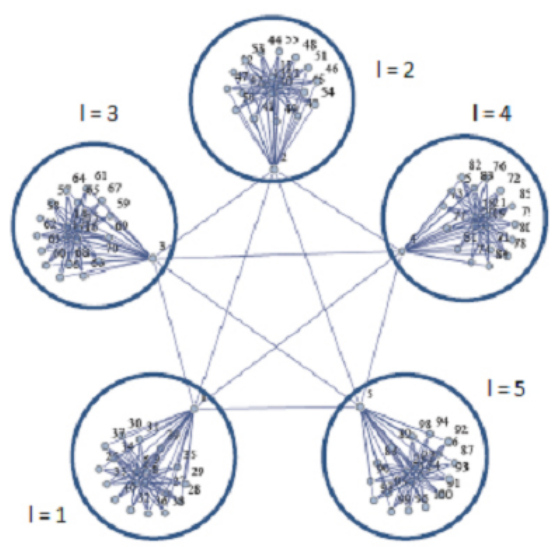

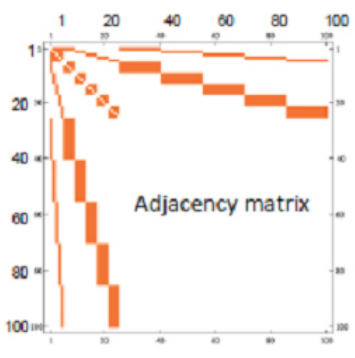

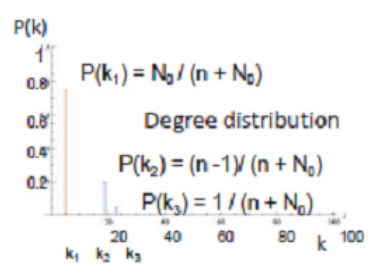

Number of total nodes: $N=N^{(1)}=n\left(n+N_{0}\right) ; \quad I=n, N_{0} \geqq 0$
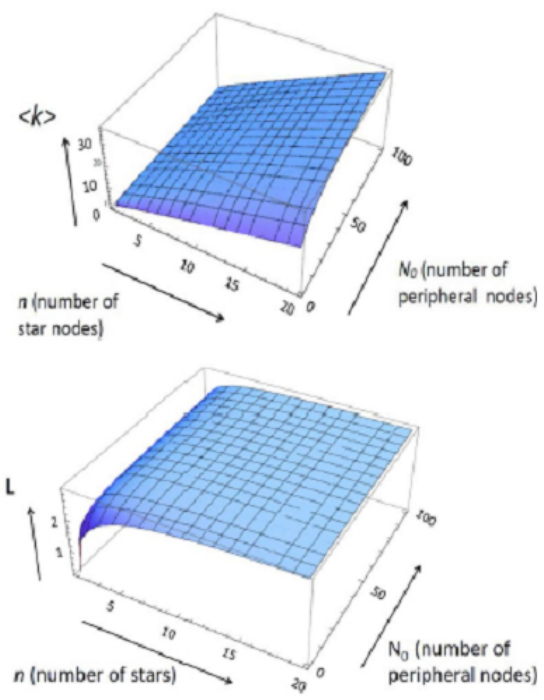

$n$ : Number of star nodes, I: layer number of hierarchy, No: Number of peripheral nodes.

Average degree: $\left\langle k^{(1)}\right\rangle=\left[n^{2} N_{0}+n(n-1)\left(n+N_{o}-1\right)+\left\{2(n-1)+N_{0}\right\} n\right] / N$

Average path length:

$$
\begin{aligned}
L^{(I)}= & {\left[{ }_{n} C_{2}+n_{n-1} C_{2}+2 n_{N_{0}} C_{2}+n(n-1)(2 n-1)+3(n-1)^{2}{ }_{n} C_{2}+n(n-1) N_{O}\right.} \\
& \left.+3 n(n-1)^{2} N_{O}+n(2 n-1) N_{O}+3 n{ }_{n} C_{2} N_{O}{ }^{2}\right] /{ }_{N} C_{2}
\end{aligned}
$$

Clustering coefficient:

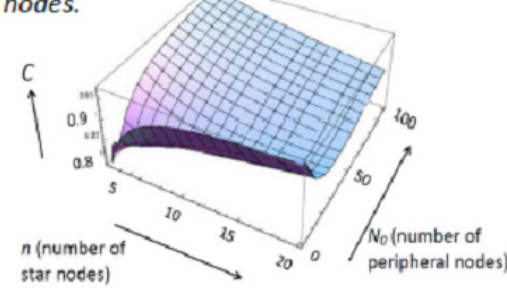

$C^{(I)}=\left[n\left\{2_{n-1} C_{2}+(n-1) N_{0}\right\} / 2(n-1)+N_{0} C_{2}+n(n-1)\left\{n-1 C_{2}+(n-1) N_{0}\right\} /{ }_{n-1+N_{0}} C_{2}+n N_{0}\right] / N$

Figure 7: Schematic diagram of the fractal type II (top-left), its adjacency matrix (top-middle), degree distribution $P(k)$ (middle), the 3D viewgraphs of average degree $\langle k\rangle$ (top-right), average path-length $L$ (middle-right) and clustering coefficient $C$ (bottom-right), and analytical results on several network parameters such as average degree $\langle k\rangle$, average path-length $L$ and clustering coefficient $C$ (bottom-left) are shown.

\subsection{Analysis of assotativity $r$}

tativity $r$ is defined ${ }^{[6]}$ as follows :

We newly analyze the degree correlation. The degree correlation is called "assortativity" in complex networks. Assor-

$$
r=\frac{\sum_{(u, v) \in E}^{M} k_{u} k_{v}-M^{-1}\left(1 / 2 \sum_{(u, v) \in E}^{M}\left(k_{u}+k_{v}\right)\right)^{2}}{1 / 2 \sum_{(u, v) \in E}^{M}\left(k_{u}^{2}+k_{v}^{2}\right)-M^{-1}\left(1 / 2 \sum_{(u, v) \in E}^{M}\left(k_{u}+k_{v}\right)\right)^{2}},
$$

where $M$ is the total number of links, $k_{u}$ and $k_{v}$ are degrees at node $u$ and node $v$, respectively. Assortativity is a measure of how much two degrees at two adjacent nodes are similar to each other; for examples, when a hub node tends to be adjacent to another hub node, or a node with a few degrees tends to be adjacent to another node with a few degrees, the network is assortative (i.e. positively correlative); on the other hand, a hub node tends to be adjacent

Published by Sciedu Press to a node with a few degrees, and vice versa, the network is disassortative (i.e. negatively correlative). We calculated the assortativity $r$ for all kinds of the hierarchical n-Star networks with the relationship of $(n, l)$, as shown in Figure 9, as well as in Table 1 (the rightmost column) for the typical set of network parameters.

According to Table 1, the n-Star network is strongly disassortative (negatively correlative). Among the six hierar- 
chical networks except the n-Star network, the fractal type torus type are intermediately disassortative. The star type, II is the most disassortative. The hierarchical type and the star-torus type and fractal type I are the least disassortative.

Table 1: Several network parameters such as $\langle k\rangle, L, R, C$ and $r$ for all types of the hierarchical structures for a typical set of parameters such as $n, N_{0}, l$ and $N$, where $n$ is the number of star nodes, $N_{0}$ is the number of peripheral nodes, $l$ is the level of hierarchy, $N$ is the total number of nodes, $\langle k\rangle$ is average degree, $L$ is average path-length, $R$ is the degree of small-world, $C$ is clustering coefficient and $r$ is assortativiry.

\begin{tabular}{llllllllll}
\hline Network & $\boldsymbol{n}$ & $\boldsymbol{N}_{\mathbf{0}}$ & $\boldsymbol{I}$ & $\boldsymbol{N}$ & $<\boldsymbol{k}>$ & $\boldsymbol{L}$ & $\boldsymbol{R}$ & $\boldsymbol{C}$ & $\boldsymbol{r}$ \\
\hline n-Star NW & 3 & 97 & 1 & 100 & 5.88 & 1.94 & 2.0 & 0.971 & -0.980 \\
Hierarchy & 3 & 10 & 10 & 103 & 6.41 & 4.82 & 4.88 & 0.801 & -0.476 \\
Torus & 3 & 10 & 10 & 100 & 6.60 & 3.93 & 4.00 & 0.794 & -0.467 \\
Star & 3 & 7 & 10 & 100 & 6.42 & 3.04 & 3.11 & 0.803 & -0.081 \\
Star-Torus & 3 & 7 & 10 & 100 & 8.04 & 2.86 & 2.94 & 0.814 & -0.139 \\
Fractal I & 3 & 7 & 10 & 100 & 9.12 & 2.74 & 2.83 & 0.828 & -0.129 \\
Fractal II & 5 & 15 & 5 & 100 & 8.70 & 2.64 & 2.73 & 0.841 & -0.704 \\
\hline
\end{tabular}

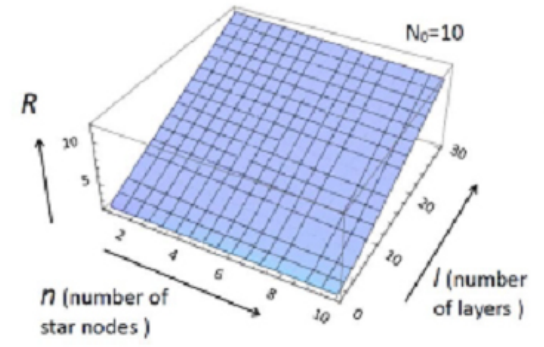

(a) Hierarchical type

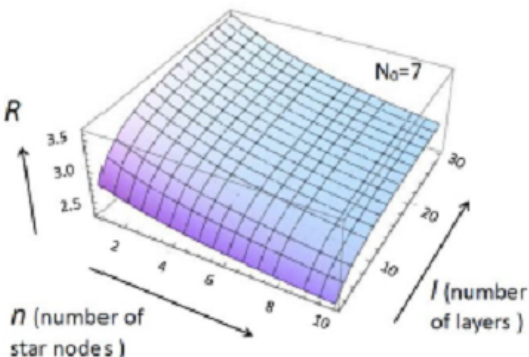

(c) Star type

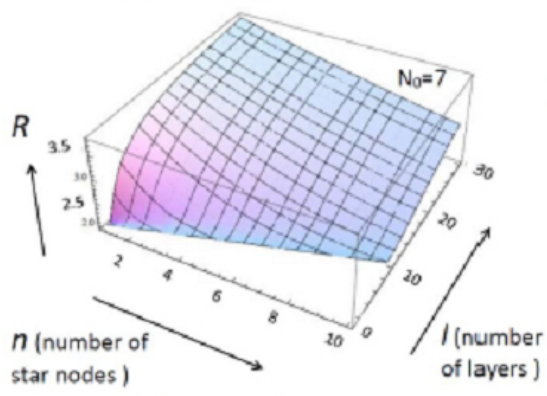

(e) Fractal type I

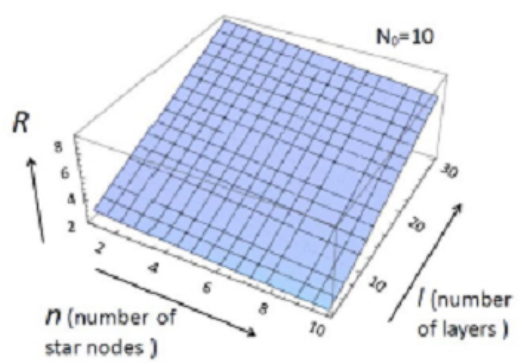

(b) Torus type

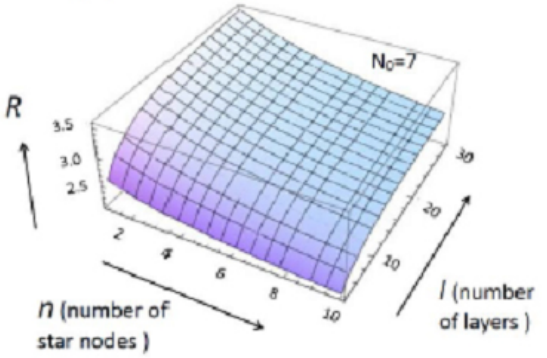

(d) Star-Torus type

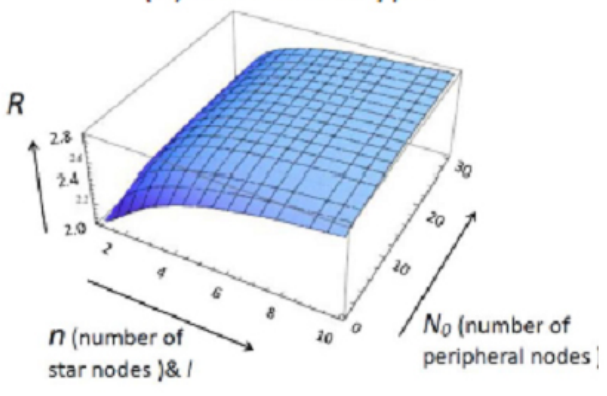

(f) Fractal type II

Figure 8: Analysis of the small-world index $R$ for several hierarchical structures of n-Star networks. 


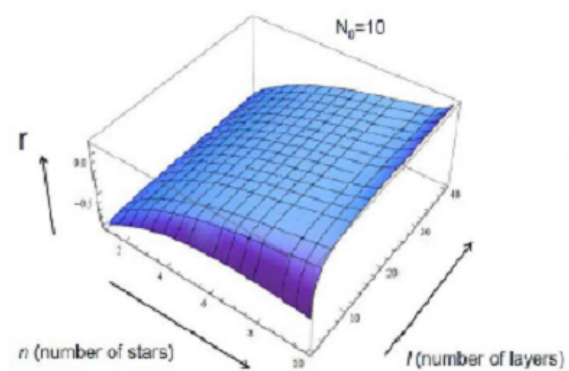

(a) Hierarchical type

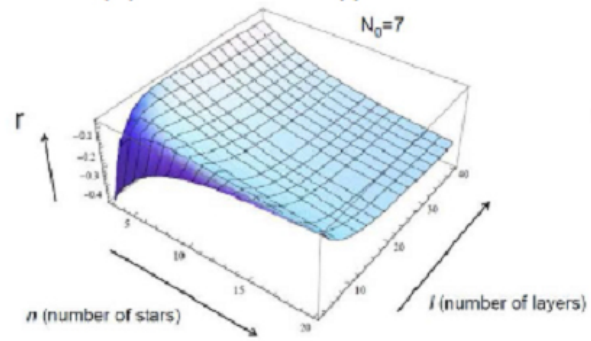

(c) Star type

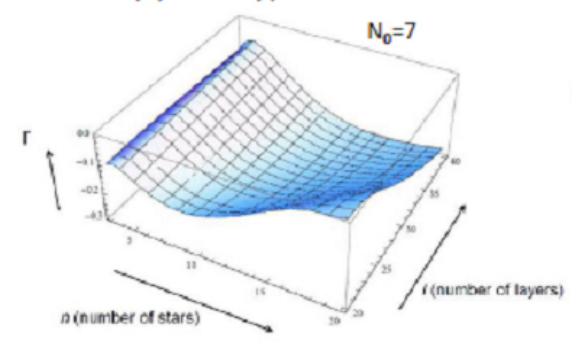

(e) Fractal type I

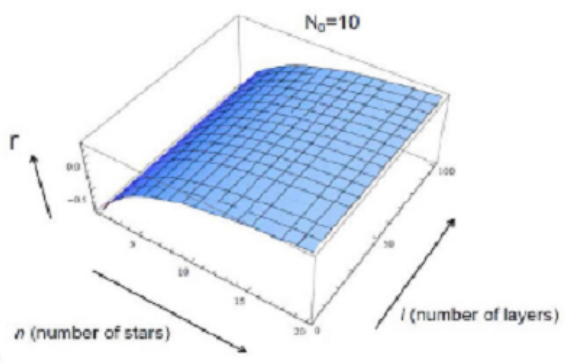

(b) Torus type

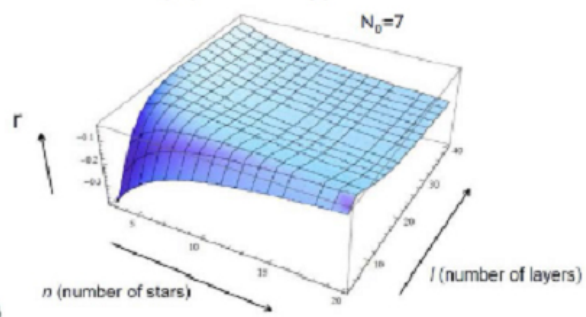

(d) Star-Torus type

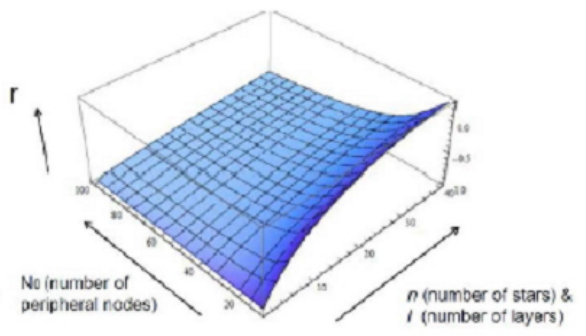

(f) Fractal type II

Figure 9: Analysis of assortativity $r$ for several hierarchical structures of n-Star Networks

\section{Application to hierarchical structure of global airline networks}

We consider an airline network using the fractal type II as an example of real-world applications of the hierarchical nStar networks. The characteristic that an airline network should meet is as follows: A current world airline network is formed around hub airports. It is thought that a world hub airport is formed based on the relationships such as the geographical position (latitude, longitude, altitude on each continent to which each city belongs), population, importance in the economic aspect and geographical distance (distance along the geodesic line) from the other hub airports. In other words, there are more flights between hub airports and their outskirts cities with "preferential attachment", and it is well known to be a "scale-free network" having degree distribution with power-law, which is often modelled by the BA model. ${ }^{[22]}$

When a direct route linking between a hub airport and its outskirts airports exists, a current airline network is very convenient. On the other hand, when a direct route linking between a hub city and its outskirts airports doesn't exist, it is not so convenient because the number of transits would increase at the hub airport.

Published by Sciedu Press
Therefore, there are two different strategies between Boeing and Airbus companies (both are two major airplane vendors in the world) to deal with above situation; the former company (Boeing) takes a strategy to use mid-sized airplanes for connecting airports directly with each other, which is called the "Point-to-Point" strategy. On the other hand, the latter company (Airbus) takes a strategy to connect hub airports with each other using big airplanes like jumbo jets, and then to transit to their neighboring airports using small airplanes, which is called the "Hub \& Spoke" strategy.

The "Point-to-Point" strategy is superior from a viewpoint of direct access flight, but there is room for improvement about the small-world characteristics as a whole network. On the other hand, the "Hub \& Spoke" strategy is superior from a viewpoint of the small-world characteristics, but it is inferior to the "Point-to-Point" strategy with direct access. Therefore, both strategies have both merit and demerit.

The airline network based on the n-Star network is close to the "Hub \& Spoke" strategy from the viewpoint of having the star airports with their peripheral airports, and is also close to the "Point-to-Point" strategy from the viewpoint of having peripheral links. Therefore, the n-Star-based airline network has both merits of the two different strategies. 
It is necessary for neighboring airports on each continent to link with star airports in other continents if an airline network is based on the n-Star network, but a local airport on a continent usually tends to connect with another local airport on another continent through its nearest hub airport on the same continent. Therefore, it would naturally form a hierarchical structure of network when the number of airports increases in the world.

\section{Experimental results and discussions}

According to the small-world characteristics based on the $R$ values, the airline network can be formed, as shown in Figure 10 , based on the fractal type II with the best value of $R$ among the various hierarchical types of networks in Table 1 . The peripheral links are formed based on the importance between airports, i.e. the n-Star network in the fractal type II is equivalent to the n-Star network with neighboring links as denoted by dotted lines in Figure 1 (left). The top 100 ranking cities in the world are extracted from the city database. ${ }^{[34]}$ As five star airports, Tokyo, Chicago, Frankfurt, Sydney and Johannesburg are selected from each continent, and the n-Star network structure is also formed on each continent around each star airport. The average degree $\langle k\rangle$ is 15.5 which corresponds to the average number of different flight routes per airport. The average pathlength $L$ (dist) is 1.410 which corresponds to about 8,980 $\mathrm{km}$ in the unit of radius of Earth, about $6,368 \mathrm{~km}$. The average number of transits is 1.421 because the average path-length $L$ (step) is 2.421 which corresponds to the number of transits plus one. The $R($ step $)$ value calculated by $R($ step $) \equiv L($ step $)+p=L($ step $)+\langle k\rangle /(N-1)$ is 2.577 . These values are summarized in Table 2 with comparison of the networks based on the scale-free network (BA model) and n-Star network. In comparison with the current scalefree network with almost the same value of $\langle k\rangle$, the value of $L$ (dist) is smaller by $9.3 \%$ than that of the scale-free network while the value $L(s t e p)$ is close to that of the scalefree network, i.e. the small-worldness $R$ is as good as that of the current scale-free network. Also, in comparison with the basic n-Star network with almost the same value of $\langle k\rangle$, although the value of $L$ (step) increases by $27.0 \%$, the value of $L$ (dist) slightly decreases by $0.84 \%$, which may be considered as the merit of hierarchical structure of n-Star network.

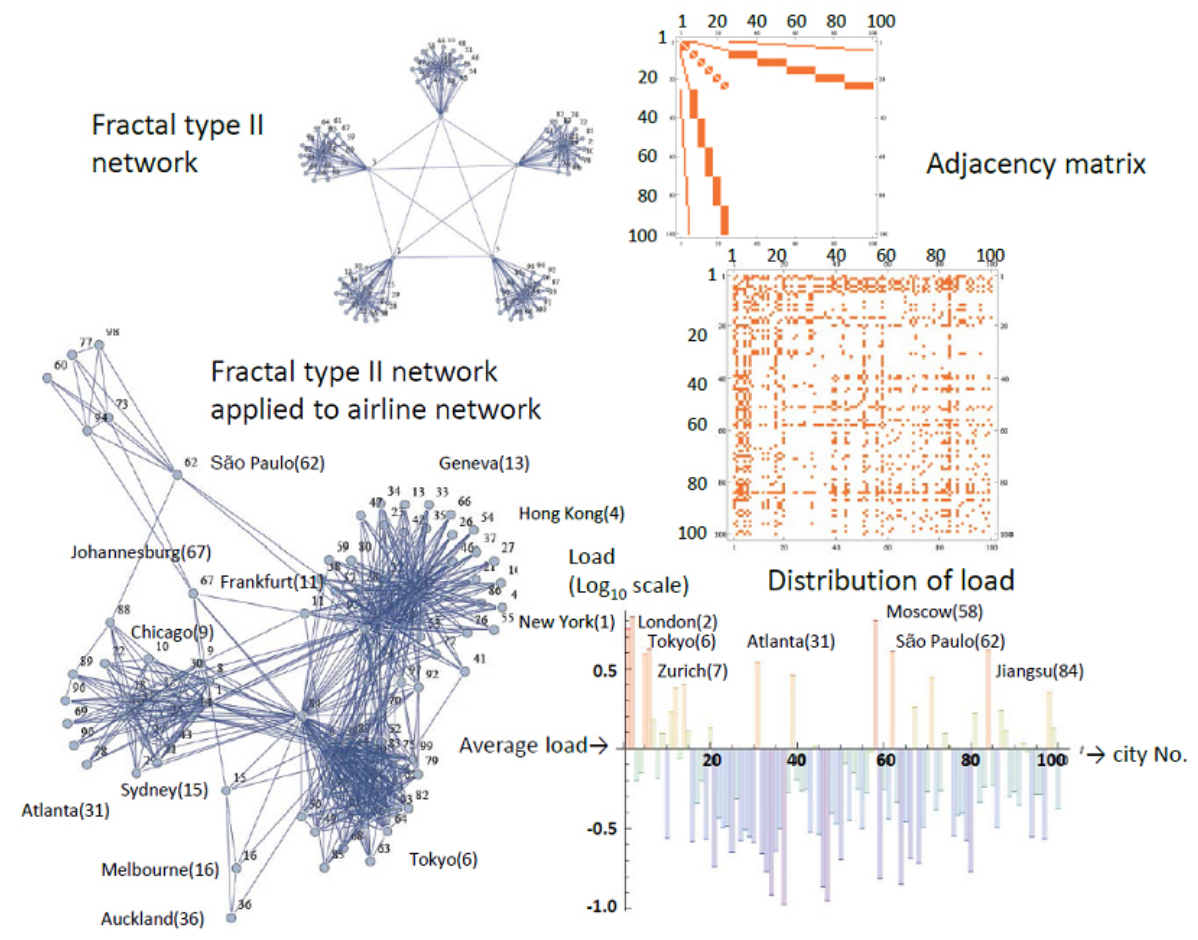

Figure 10: A global airline network based on the fractal type II. For reference, the original fractal type II of n-Star network in Figure 7 is also shown in the upper region of this figure. As five star airports, Tokyo (No.6), Chicago (No.9), Frankfurt (No.11), Sydney (No.15) and Johannesburg (No.67) are selected from the top 100 ranking cities ${ }^{[34]}$ on each continent, and each n-Star network structure is formed around each star airport on each continent. The load concentration on the star airports and some specific airports are moderately distributed, as shown in the bottom-right region of this figure. 
In summary, the airline network based on the fractal type II is advantageous in terms of $L($ dist $)$ compared to the current scale-free (the BA model) network and the basic n-Star network with the comparable number of transits $L(s t e p)$ and the small-worldness $R($ step $)$ of the current scale-free network. The difference in $L$ (dist) between the fractal type II and the scale-free networks is significantly large because the travel time as well as the jet fuel consumption in proportion to the average distance $L(d i s t)$ will be reduced while keeping the same convenience (i.e. $L($ step $)$ ) as the current airline network.

Generally speaking, there is a tendency of concentrating loads on star airports if we design a star type airline network. However, the loads of concentration on the star airports in the fractal type II airline network are moderately distributed, as shown in Figure 10 (bottom-right) where the loads of several airports such as New York (No.1), London (No.2), Tokyo (No.6), Zurich (No.7), Atlanta (No.31), Moscow (No.58), São Paulo (No.62) and Jiangsu (No.84) are controlled under less than 10 times at maximum of the average loads by 100 airports.

Table 2: Comparison of performance in global airline networks based on different types of networks

\begin{tabular}{llll}
\hline $\begin{array}{l}\text { Network/para- } \\
\text { meter }\end{array}$ & $\begin{array}{l}\text { Fractal type II } \\
\text { network }\end{array}$ & $\begin{array}{l}\text { Scale-free } \\
\text { (BA model) }\end{array}$ & $\begin{array}{l}\text { n-Star } \\
\text { network }\end{array}$ \\
\hline$<k>$ & 15.5 & 15.41 & 15.52 \\
L(dist) & 1.410 & 1.516 & 1.422 \\
L(step) & 2.421 & 2.417 & 1.907 \\
R(step) & 2.577 & 2.573 & 2.064 \\
\hline
\end{tabular}

\section{Conclusions}

We have extended the n-Star network to several hierarchical structures and analyzed the features of each network theo- retically using network parameters such as the total number of nodes, average degree, average path-length, degree distribution and assortativity (degree correlation), etc. Also, we defined a degree of small-world, called "small-worldness" which is a simple measure of linear combination of average path-length (corresponding to efficiency of network topology) with link probability (corresponding to cost of network). Using this measure, any types of networks including hierarchical types are evaluated in terms of network topology. As the n-Star network is proved to be the smallest-world in this sense having the minimum value of small-worldness 2 while the other networks can be evaluated how far their values are from the minimum value of 2. Among several types of the hierarchical structures of nStar network, we found that the fractal type II is the best in this sense. Based on this knowledge, the fractal type II of n-Star network-based world airline network architecture was proposed, and simulation experiments were performed with comparison of the current airline network and the basic n-Star network-based airline network. As a result, the proposed hierarchical structure is more suitable for real world because the shorter average cruising distance than the other two kinds of networks is realized with the comparable number of transits to the current airline network with scale-free property. Future studies will focus on application of the proposed hierarchical architectures of n-Star network to the fields of communication networks, logistics networks, utility networks and power networks, etc. in the real world.

\section{Acknowledgements}

The author would like to thank the anonymous reviewers for their valuable suggestions and comments.

\section{References}

[1] Watts J.D. Small Worlds, The Dynamics of Networks between Order and Randomness, Princeton University Press, 1999.

[2] Sawai H. Exploring a New Small-World Network for Real-World Applications, Proc. of the 4th Int. Conf. on Networked Digital Technology (NDT 2012), Dubai, UAE, Apr. 2012.

[3] Sawai H. Reorganizing A New Generation Airline Network Based on An Ant-Colony Optimization-Inspired Small-World Network, 2012 IEEE World Congress on Computational Intelligence (WCCI 2012), Brisbane, Australia, June 2012.

[4] Sawai H. A Small-World Network Immune from Random Failures and Resilient to Targeted Attacks, Proceedings of the International Conference on Computational Science (ICCS 2013), pp.976-985, Barcelona, Spain. June 2013: 976-985.

[5] Sawai H. Structuring Hierarchical Multi-Star Small-World Networks for Real-World Applications, Int. Conf. on Computational Science (ICCS 2013). June 2013: 2410-2419, Barcelona, Spain.

[6] Newman M.E.J. Assortative Mixing in Networks, Phys. Rev. Lett. $2002 ; 89(11): 1-4$
[7] Comellas F., Fertin G., Raspaud A. Recursive graphs with smallworld scale-free properties, Phys. Rev. E. 2004; 69: 1-4.

[8] Zhang Z., Rong L., Zhou S. Evolving Apollonian networks with small-world scale-free topologies, Phys. Rev. E. 2006; 74: 1-9.

[9] Klemm K., Eguíluz V.M. Growing scale-free networks with smallworld behavior, Phys. Rev. E. 2002; 65: 1-4.

[10] Ping X., Feng L., Wei L. Growing small-world networks based on a modified BA model, Chinese Physics Letters. 20006; 23(3): art. no. 750 .

[11] Giabbanelli P.J. The Small-world properties in networks growing by active edges, Advances in Complex Systems. 2011; 14(6): 853-869, World Scientific Publishing Company.

[12] Shang Y. Distinct clusterings and characteristic path lengths in dynamic small-world networks with identical limit degree distribution, Journal of Statistical Physics. 2012; 149(3): 505-518. http: //dx.doi.org/10.1007/s10955-012-0605-8

[13] Shang Y. Geometric Assortative Growth Model for Small-World Networks, The Scientific World Journal. 2014: 1-8.

[14] Masuda N., Miwa H., Konno N. Geographical threshold graphs with small-world and scale-free properties, Phys. Rev. E. 2005; 71: 1-10. 
[15] Hoore M., Moghimi-Araghi S. Critical behavior of a small-world sandpile model. Journal of Physics A. 2013; 46: art. no. 195001.

[16] Shang Y. A sharp threshold for rainbow connection in small-world networks, Miskolc Mathematical Notes. 2012; 13(2): 493-497.

[17] Mathias N., Gopal V. Small worlds: How and why, Phys. Rev. E. 2001; 63: 021117. http://dx.doi.org/10.1103/PhysRevE. 63.021117

[18] Watts D.J., Strogatz S.H. Collective dynamics of 'small-world' networks. Nature. 1998; 393(4): 440-442.

[19] Nishikawa T., Motter A.E., Lai Y-C., Hoppensteadt F.C. Smallest small-world network, Phys. Rev. E. 2002; 66: 1-5

[20] Tang J., Scellato S., Musolesi M., Mascolo C., Latora V. Smallworld behavior in time-varying graphs, Phys. Rev. E. 2010; 81: 1-4.

[21] Humphries M.D., Gurney K., Prescott T.J. The braistem reticular formation is a small-world, not scale-free, network. Proceedings of the Royal Society B. 2006; 273: 503-511. http://dx.doi.org/ 10.1098/rspb.2005.3354

[22] Barabási A-L.\& Albert R. Emergence of Scaling in Random Networks. Science. 1999: 509-512.

[23] Bollobás B., Riordan O. The diameter of a scale-free random graph, Combinatorica. 2004; 24: 5-34. http://dx.doi.org/10.1007/ s00493-004-0002-2

[24] Rosenfeld H.D. Havlin S., ben-Avraham D. Fractal and Transfractal Recursive Scale-Free Nets, New Journal of Physics. 2007; 9: article No.175.
[25] Song C., Havlin S., Makse H.A. Self-similarity of complex networks, Nature. 2005; 433: 392-395. http://dx.doi.org/10. 1038 /nature03248

[26] Song C., Havlin S., Makse H.A. Origins of fractality in the growth of complex networks. Nature Physics. 2006; 2: 275-281. http: //dx.doi.org/10.1038/nphys266

[27] Barabási A.-L., Ravasz E., Vicsek T. Deterministic scale-free networks, Physica A. 2001; 299: 559-564.

[28] Dorogovtsev S.N., Goltsev A.V., Mendes J.F.F. Pseudofractal scalefree web, Phys. Rev. E. 2002; 65: article No.066122.

[29] Ravasz E., Somera A.L., Mongru D.A., Oltvai Z.N., Barabási A.L. Hierarchical Organization of Modularity in Metabolic Networks, Science. 2002; 297: 1551-155.

[30] Ravasz E., Barabási A.-L. Hierarchical organization in complex networks, Phys. Rev. E. 2003; 67: article No.026112.

[31] Yakubo K., Kurošak D. Scale-free networks embedded in fractal space, Phys. Rev. E. 2011; 83: 1-11.

[32] Chelminiak P. Emergence of fractal scale-free networks from stochastic evolution on the Cayley tree, Physics Letters A. 2013; 377: 2846-2850. http://dx.doi.org/10.1016/j.physleta. 2013.08 .042

[33] Shang Y. Mean commute time for random walks on hierarchical scale-free networks. Internet Mathematics. 2012; 8(4): 321-337.

[34] Hot Spots. Benchmarking Global City Competitiveness, A Report from the Economist Intelligence Unit, Commissioned by Citi. American Psychological Association. Jan. 2012. 